\title{
Mammalian Spermidine Synthase-Identification of Cysteine Residues and Investigation of the Putrescine Binding Site-
}

\author{
Hitomi Goda, Toshiko Watanabe, Noboru Takeda, Masaki Kobayashi, Makiko Wada, \\ Harumi Hosoda, Akira Shirahata, and Keijiro SAMEJIMA* \\ Faculty of Pharmaceutical Sciences, Josai University; 1-1 Keyakidai, Sakado, Saitama 350-0295, Japan. \\ Received May 10, 2004; accepted July 2, 2004
}

\begin{abstract}
Homology modeling and inhibitory studies using substrate analogs were undertaken to construct a possible three-dimensional structure, including the putrescine-binding site, of rat spermidine synthase based on its primary sequence. Of the ten cysteine residues of the enzyme, six residues were chemically determined as sulfhydryl; similarly, one residue (C25) was determined as the disulfide. Using the model obtained from the Swiss-Model protein-modeling server, and based on the crystal structure of the Thermotoga maritima enzyme, the three remaining residues were assigned as sulfhydryl. Discussions are presented on the counterpart of the $\mathrm{C} 25$ residue, based on the apparent role of the bacterial $\mathrm{N}$-terminal peptide region in reinforcing the binding between protomers in a functional oligomeric form. The active sites of the bacterial and mammalian versions of the enzyme were very similar. The putrescine-binding site of the rat enzyme was investigated using IC $_{50}$ values of the analogs of two known potent inhibitors, $n$-butylamine and trans-4-methylcyclohexylamine (4MCHA). Our results indicated that 5-amino-1-pentene and 4MCHA possess comparable inhibitory activities towards the enzyme.
\end{abstract}

Key words putrescine aminopropyltransferase; homology modeling; inhibitor; trans-4-methylcyclohexylamine; 5-amino-1-pentene; matrix assisted laser desorption ionization time-of-flight mass spectrometer (MALDI-TOF-MS)

Spermidine synthase (spd syn, putrescine aminopropyltransferase) has been shown to catalyze the transfer reaction of the aminopropyl moiety of decarboxylated $S$-adenosyl-Lmethionine (dcAdoMet) into putrescine in the formation of spermidine, which is widely distributed among living organisms with increasing levels related to cell growth. ${ }^{1-3)}$ In addition to the reported amino acid sequence of spd syn from various sources, ${ }^{4,5)}$ the crystal structures of some of these bacterial enzymes have been recently reported ${ }^{6}$ (PDB accession codes 1INL, 1JQ3, 1MJF, 1IY9, and 1UIR). One of the significant differences between the amino acid sequence of mammalian and bacterial enzymes is the content of cysteine residue, i.e. ten residues for mammalian enzymes, three for Thermotoga maritima, and none for Bacillus subtilis and Pyrococcus furiosus. Accordingly, the determination of each cysteine residue as either sulfhydryl or disulfide is essential for the accurate homology modeling of mammalian spd syn. The primary sequence of rat spd syn is shown in Fig. 1. ${ }^{7}$

The use of specific inhibitors is another approach in the study of the substrate-binding site. Using a number of monoamine and diamine compounds, we have proposed a model of the putrescine-binding site of pig spd syn, ${ }^{8)}$ which features a relatively large hydrophobic cavity adjacent to a negatively charged site. Presumably, one of the amino group of putrescine is protonated and binds to this charged site, and the other amino group is not protonated and binds to the hydrophobic cavity, to be aminopropylated by dcAdoMet. The

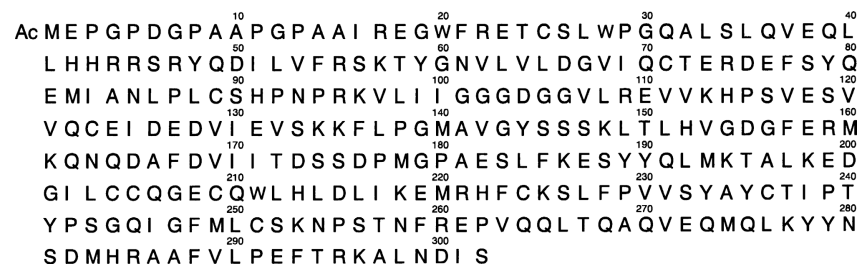

Fig. 1. Primary Structure of Rat Spermidine Synthase substrate-binding site of our model shows good agreement with that of the crystal structure of the T. maritima enzyme. ${ }^{6}$

The present study was undertaken to identify the ten cysteine residues of rat spd syn exist as sulfhydryl or disulfide, and to investigate the putrescine-binding site using several compounds that are based on two known potent inhibitors for mammalian spd syn, trans-4-methylcyclohexylamine (4MCHA) and $n$-butylamine (BA). Based on the results, a three-dimensional model for mammalian spd syn was proposed using the homology modeling approach based on the crystal structure of the T. maritima enzyme.

\section{MATERIALS AND METHODS}

Chemicals Dithiothreitol (DTT), guanidine hydrochloride, monoiodoacetic acid (MIA), trans-4-hydroxycyclohexyl amine (4HCHA), 5-[ $N$-(iodoacetamidoethyl)amino]naphthalene-1-sulfonic acid (IAEDANS), 2-nitro-5-thiocyanobenzoic acid (NTCB), and tris(2-carboxyethyl)phosphine hydrochloride (TCEP) were purchased from Sigma. Decarboxylated $S$-adenosylmethionine (dcAdoMet) was prepared in our laboratories. ${ }^{9)} \mathrm{N}$-Hydroxysuccinimidyl bromoacetate (HSBA) was also prepared in our laboratories using bromoacetic acid, $N$-hydroxysuccinimide, and dicyclohexylcarbodiimide. $n$-Propylamine, $n$-butylamine (BA), n-pentyl amine (PA), $n$-hexylamine (HA), and cyclohexylamine (CHA) were purchased from Tokyo Kasei Kogyo Co. Ltd., and were recrystallized as their hydrochlorides. 4-Methylcyclohexylamine (as a mixture of cis- and trans-isomers) was purchased from Sigma, and was repeatedly recrystallized as the hydrochloride to afford pure trans-4-methylcyclohexylamine hydrochrolide (4MCHA), as confirmed by NMR. All other reagents and organic solvents were of commercial analytical grade.

BA Analogs 4-Bromo-1-butene (Acros) was subjected to reaction with potassium phthalimide in DMF, followed by 
hydrazine degradation, ${ }^{10)}$ then recrystallized from ethyl acetate and $\mathrm{EtOH}$ to afford 4-amino-1-butene hydrochloride (ABE; Anal. Calcd for $\mathrm{C}_{4} \mathrm{H}_{10} \mathrm{NCl}$ : C, 44.66; H, 9.37; N, 13.02. Found: C, 42.36; H, 9.20; N, 13.55). Similarly, 5bromo-1-pentene and 6-bromo-1-hexene were used to afford 5-amino-1-pentene hydrochloride (APE; Anal. Calcd for $\mathrm{C}_{5} \mathrm{H}_{12} \mathrm{NCl}: \mathrm{C}, 49.38 ; \mathrm{H}, 9.55 ; \mathrm{N}, 11.52$. Found: $\mathrm{C}, 49.15 ; \mathrm{H}$, 10.14; N, 11.52) and 6-amino-1-hexene hydrochloride (AHE; Anal. Calcd for $\mathrm{C}_{6} \mathrm{H}_{14} \mathrm{NCl} \cdot 0.1 \mathrm{H}_{2} \mathrm{O}: \mathrm{C}, 52.43 ; \mathrm{H}, 10.41 ; \mathrm{N}$, 10.19. Found: C, 52.40; H, 10.38; N, 10.14), respectively. 5Amino-1-pentyne hydrochloride (APYN; Anal. Calcd for $\mathrm{C}_{5} \mathrm{H}_{10} \mathrm{NCl}$ : C, 50.22; H, 8.43; N, 11.71. Found: C, 49.99; H, 8.46; N, 11.71) was prepared from 4-pentyn-1-ol (Acros) according to the reported method. ${ }^{11)}$ 6-Amino-1-hexyne hydrochloride (AHYN; Anal. Calcd for $\mathrm{C}_{6} \mathrm{H}_{12} \mathrm{NCl}$ : C, 53.93; H, 9.05; N, 10.48. Found: C, 53.85; H, 9.14; N, 10.54) was prepared via hydrazine degradation of 6-phthalimide-1-hexyne (Aldrich).

4MCHA Analogs 4,4-Dimethyl-2-cyclohexene-1-one (Aldrich) was reduced to 4,4-dimethyl-2-cyclohexanone, ${ }^{12}$ ) derivatized to 4,4-dimethyl-2-cyclohexaneoxime, ${ }^{13)}$ then reduced with sodium bis(2-methoxyethoxy)aluminium hydride to afford 4,4-dimethylcyclohexylamine hydrochloride (DMCHA; Anal. Calcd for $\mathrm{C}_{8} \mathrm{H}_{18} \mathrm{NCl}$ : C, 58.70; H, 11.08; N, 8.56. Found: C, 58.49; H, 11.08; N, 8.61). 1,4-Cyclohexanedione monoethylene ketal (Aldrich) was reacted with methylenetriphenylphosphorane to form 4-methylenecyclohexyl monoethylene ketal, ${ }^{14)}$ the deprotected 4-methylenecyclohexanone was subjected to similar treatments as described for DMCHA to afford 4-methylenecyclohexylamine hydrochloride (MLCHA; Anal. Calcd for $\mathrm{C}_{7} \mathrm{H}_{14} \mathrm{NCl}$ : C, 56.94; H, 9.56; N, 9.49. Found: C, 56.69; H, 9.72; N, 9.44). trans-4Ethylcyclohexylamine hydrochloride (4ECHA; Anal. Calcd for $\mathrm{C}_{8} \mathrm{H}_{18} \mathrm{NCl}$ : C, 58.70; H, 11.08; N, 8.56. Found: C, 58.68; $\mathrm{H}, 11.03 ; \mathrm{N}, 8.37$ ) was prepared from 4-ethylcyclohexanone (Aldrich) following similar methods as described for DMCHA.

Purification and Assay of Spd Syn Spd syn was obtained from the ventral prostate of male Sprague Dawley rats and purified to homogeneity following a previously reported method. ${ }^{15)}$ Purified spd syn was stocked in $25 \mathrm{~mm}$ sodium phosphate ( $\mathrm{pH} 7.2)$ containing $0.3 \mathrm{~mm}$ EDTA and $0.5 \mathrm{~mm}$ DTT (Buffer A), $0.3 \mathrm{M} \mathrm{NaCl}$, and $1 \mathrm{~mm}$ dcAdoMet (about $230 \mu \mathrm{g} / \mathrm{ml})$ at $0{ }^{\circ} \mathrm{C}$.

Spd syn activity was measured using [S,R-methyl$\left.{ }^{14} \mathrm{C}\right] \mathrm{dcAdoMet}{ }^{9)}$ according to Hibasami and Pegg. ${ }^{16}$ The standard assay medium contained $10 \mu \mathrm{M}$ dcAdoMet, $1 \mathrm{~mm}$ putrescine, $5 \mathrm{~mm}$ DTT, $0.75 \mathrm{mg} / \mathrm{ml}$ bovine serum albumin, and $0.1 \mathrm{M}$ potassium phosphate $(\mathrm{pH} 7.4)$. Inhibition studies were carried out by measuring spd syn activities in the presence of the tested compounds with concentrations of 0 to $1.0 \mathrm{~mm}$.

Chemical Cleavage of Spd Syn at the Cysteine Residue $^{17)}$ To about $0.15 \mathrm{nmol}$ of HSBA-modified spd syn $(50 \mu \mathrm{l})$ was added $0.1 \mathrm{M}$ sodium phosphate $(\mathrm{pH} 8.0)(120 \mu \mathrm{l})$ containing $8 \mathrm{M}$ guanidine hydrochloride (Buffer B) and $1.3 \mathrm{~mm}$ TCEP, followed by the addition of an $18 \mu \mathrm{l}$ aliquot of a mixture of Buffer A (1 volume) containing $0.01 \mathrm{M} \mathrm{NTCB}$ and Buffer B (3 volumes). The mixture was incubated at $37^{\circ} \mathrm{C}$ for $30 \mathrm{~min}$. After adjusting to $\mathrm{pH} 9$ using $1.5 \mathrm{~m}$ sodium hydroxide $(3 \mu \mathrm{l})$, the mixture was incubated at $37^{\circ} \mathrm{C}$ for $16 \mathrm{~h}$.
Enzymatic Cleavage (Trypsin) Digestion using trypsin (Promega, sequencing grade) was carried out in 44\% acetonitrile according to literature. ${ }^{18)}$ Pretreatment of spd syn was as follow: to a stock solution of spd syn $(50 \mu \mathrm{l})$, was added $0.67 \mathrm{M}$ Tris- $\mathrm{HCl}(\mathrm{pH} 8.5,150 \mu \mathrm{l})$ containing $13.4 \mathrm{~mm}$ EDTA and guanidine hydrochloride $(133 \mathrm{mg})$. The denatured spd syn solution $(200 \mu \mathrm{l})$ was reduced using $2.5 \mathrm{~mm}$ DTT $(5.7 \mu \mathrm{l})$ for $2 \mathrm{~h}($ designated as DTT +$)$, and cysteine residues were carboxymethylated with $5 \mathrm{~mm}$ MIA $(10 \mu \mathrm{l})$ for $1 \mathrm{~h}$ at room temperature. The reaction mixture was dialyzed against $50 \mathrm{~mm}$ ammonium carbonate. The non-reduced version (designated as DTT-) was similarly prepared except for the omission of DTT-reduction.

Enzymatic Cleavage (Lysyl Endopeptidase) Digestion using lysyl endopeptidase (Achromobactor protease I, Wako Pure Chemicals, $0.15 \mu \mathrm{g} / \mu \mathrm{l}$ ) was carried out according to the literature. ${ }^{19)}$ Pretreatment of IAEDANS-labelled spd syn was as follow: dialyzed spd syn in a solution $(500 \mu \mathrm{l})$ of $6 \mathrm{M}$ guanidine hydrochloride and $10 \mathrm{~mm}$ DTT was incubated at $37^{\circ} \mathrm{C}$ for $60 \mathrm{~min}$. MIA was added to the solution (to a concentration of $20 \mathrm{~mm}$ ), and the resulting mixture was kept at room temperature for $15 \mathrm{~min}$. After the addition of 2-mercaptoethanol (to a concentration of $35 \mathrm{~mm}$ ), the reaction mixture was dialyzed against $0.05 \mathrm{M}$ Tris- $\mathrm{HCl}(\mathrm{pH} 9.0)$ containing $1 \mathrm{~mm}$ EDTA and $5 \%$ acetonitrile.

Apparatus MALDI mass spectrum was recorded in the reflector mode on a Finnigan MAT Vision 2000 TOF mass spectrometer (ThermoQuest) with a nitrogen pulse-gas laser set at a wavelength of $337 \mathrm{~nm}$. Gas-phase sequencer (Shimadzu PSQ-1) was used for the Edman method. HPLC (Shimadzu SPD-6A) conditions were as follow: column, TSK gel ODS $120 \mathrm{~T}(4.6 \mathrm{~mm}$ i.d. $\times 250 \mathrm{~mm})$; mobile phase, linear gradient from $5 \%$ to $80 \%$ acetonitrile containing $0.1 \%$ TFA; flow rate, $1 \mathrm{ml} / \mathrm{min}$; fluorescence detection, ex $350 \mathrm{~nm}$, em $500 \mathrm{~nm}$

\section{RESULTS}

Identification of the Disulfide Bond. Reaction with HSBA (Experiment 1) The use of bifunctional reagent HSBA to cross-link amino acid residues located at the surface of spd syn in the presence of dcAdoMet helped identify the disulfide bond for residue $\mathrm{C} 25$. Initially, the cross-linking reaction of spd syn was carried out at room temperature for $20 \mathrm{~min}$ in the presence of dcAdoMet with differing amounts of HSBA. Subsequently, the modified spd syn was subjected to cleavage, specifically at the cysteine residues, using NTCB following reduction with TCEP. ${ }^{17)}$ As shown in the MALDI mass spectra of the cleaved peptides (Fig. 2), increasing levels of HSBA resulted in the disappearance of a majority of the peptides, attributable to the HSBA-modification of the sulfhydryl and other functional groups. However, a major peptide ion $(m / z 2552.4)$ with a significant intensity remained unchanged, suggesting that this peptide does not contain any amino acid residues that can be modified by HSBA. Since this peptide corresponds to the N-terminus AcM1-T24 (cleaved at $\mathrm{C} 25$, Calcd $\mathrm{m} / \mathrm{z} 2552.9$ ), our experiment indicates that $\mathrm{C} 25$ initially existed as a disulfide, which is resistant to HSBA.

Comparative Studies in the Presence or Absence of DTT (Experiment 2) Following the denaturing of spd syn 


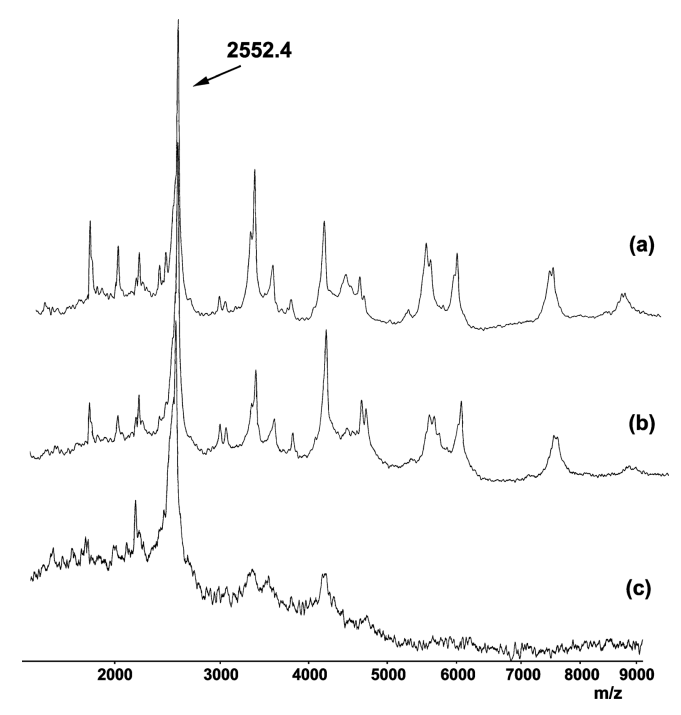

Fig. 2. MALDI Mass Spectra of NTCB-Cleaved Peptides of Spd Syn after Reaction with Different Amount of HSBA

Aliquots $(45 \mu \mathrm{l})$ of a stock solution of spd syn were reacted with dioxane solution $(5 \mu \mathrm{l})$ containing (a) 0 , (b) 25 , and (c) $100 \mathrm{nmol}$ of HSBA at room temperature for $20 \mathrm{~min}$. The reaction mixtures were then subjected to chemical cleavage at the cysteine residue as described in Materials and Methods.

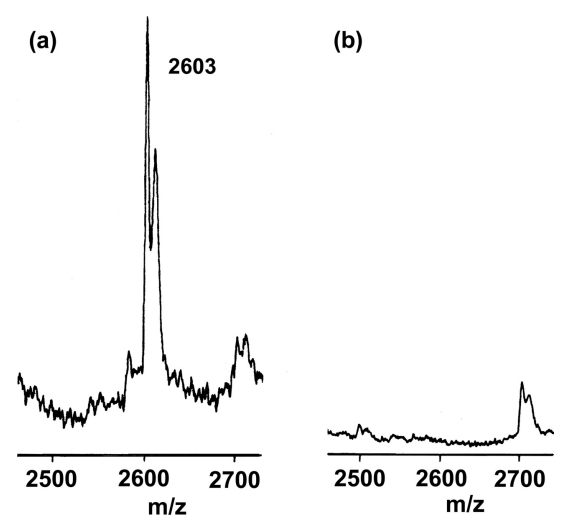

Fig. 3. MALDI Mass Spectra (Enlarged) over the Range of $m / z 2500$ 2700 in the (a) Presence and (b) Absence of DTT

Trypsin-digested sample solution $(1 \mu \mathrm{l})$ as described in Materials and Methods was added to $1 \mu \mathrm{l}$ of aqueous solution of 2,5-dihydroxybenzoic acid (DHB, $10 \mathrm{mg} / \mathrm{ml}$ ) placed on a sample target, and the mixture was allowed to air dry before being introduced into the mass spectrometer. Spectra were calibrated using DHB $(m / z 137.1)$, insulin $\left\{m / z 2867.8\right.$ for $[\mathrm{M}+2 \mathrm{H}]^{2+}, m / z 5734.6$ for $\left.[\mathrm{M}+\mathrm{H}]^{+}\right\}$, and angiotensin $\mathrm{I}(\mathrm{m} / \mathrm{z}$ 1297.4 for $\left.[\mathrm{M}+\mathrm{H}]^{+}\right)$.

using $6 \mathrm{M}$ guanidine in the presence $(\mathrm{DTT}+)$ or absence (DTT -) of DTT, the sulfhydryl groups were carboxymethylated with MIA, followed by dialysis against $50 \mathrm{~mm}$ ammonium carbonate, then subjected to trypsin digestion, as described in Materials and Methods. Among the resulting peptides, a peptide corresponding to E23-R44 (containing carboxymethylated $\mathrm{C} 25$, Calcd $\mathrm{m} / z$ 2603.3) showed a marked contrast between DTT + and DTT - in the MALDI mass spectra (Fig. 3). This results also indicated that $\mathrm{C} 25$ exists as a disulfide.

Identification of the Sulfhydryl Group. Reaction with IAEDANS (Experiment 3) To investigate the cysteine residues that are located near the active site of spd syn, the sulfhydryl groups were reacted with IAEDANS, a fluorescent $\mathrm{SH}-$ reagent. In the absence of dcAdoMet, the sulfhydryl groups were successfully labeled at $0^{\circ} \mathrm{C}$ for $30 \mathrm{~min}$, whereas,
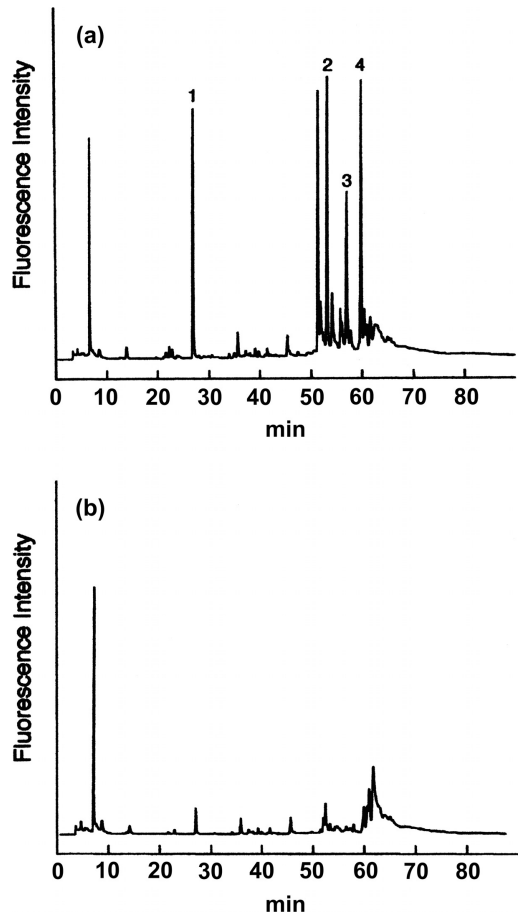

Fig. 4. HPLC of Fluorescent Labeled Peptides Obtained after Lysyl Endopeptidase Digestion of IAEDANS-Labeled Spd Syn in the (a) Absence and (b) Presence of dcAdoMet

Peak 1: E219-K225, peak 2: H114-K135, peak 3: S226-K253, peak 4: T58-K96. Aliquot of spd syn solution $(400 \mu \mathrm{l})$, after the buffer for storage was exchanged to $0.1 \mathrm{M}$ ammonium hydrogen carbonate by ultrafiltration, was subjected to $0.7 \mathrm{~mm}$ IAEDANS in the absence and presence of $0.7 \mathrm{~mm}$ dcAdoMet at $37^{\circ} \mathrm{C}$ for $2 \mathrm{~h}$, followed by $10 \mathrm{~mm}$ 2-mercaptoethanol, then dialysis against $0.25 \mathrm{~m}$ Tris- $\mathrm{HCl}(\mathrm{pH} 8.5)$. The dialyzed spd syn solutions were then digested using lysyl endopeptidase and analyzed using HPLC, as described in Materials and Methods.

in the presence of dcAdoMet, labeling was not observed. Labeled spd syn was digested with lysyl endopeptidase, and the resulting peptides were analyzed by HPLC (Fig. 4). Each fluorescent peptide was purified and applied to a gas-phase sequencer. The four major fluorescent peptides were assigned as T58-K96 (containing C71 and C89), H114-K135 (containing C123), E219-K225 (containing C224), and S226K253 (containing C236 and C251). These results indicated that $\mathrm{C} 123$ and $\mathrm{C} 224$ were sulfhydryl, and that at least one or both of C71 and C89 and of C236 and C251 was sulfhydryl.

Comparative Studies in the Presence and Absence of DTT (Experiment 4) The same experiment as described in Experiment 2 was conducted, and evidence of sulfhydryl groups for some of the cysteine residues was obtained from the MALDI mass spectra. As shown in Fig. 5A, two peptides were observed, in both DTT + and DTT - . Since each of the two peptides (T58-R74, Calcd $m / z$ 1938.0; S56-R74, Calcd $\mathrm{m} / \mathrm{z} 2153.1$ ), contain carboxymethylated $\mathrm{C} 71$, it is reasonable to suggest that C71 is sulfhydryl. In the case of Fig. $5 \mathrm{~B}$, a peptide (T195-R221, Calcd $\mathrm{m} / \mathrm{z} 3319.5$ ) was observed, in both DTT + and DTT-, indicating that the corresponding three carboxymethylated cysteine residues, C204, C205, and C209, are sulfhydryl.

The results of the above experiments helped identify seven of the ten cysteine residues of spd syn. The three remaining cysteine residues (C89, C236, and $\mathrm{C} 251)$ are possibly sulfhydryl, as described below.

Search for Putrescine Binding Site with BA and 
4MCHA Analogs Known potent inhibitors for spd syn are $S$-adenosyl-1,8-diamino-3-thiooctane (AdoDATO), ${ }^{20,21)}$ BA, and 4MCHA. To obtain information on the putrescine-binding site, analogs of BA and 4MCHA were prepared and evaluated for their inhibitory activities (Table 1). A series of BA analogs that contain a terminal double or triple bond, 4amino-1-butene (ABE), 5-amino-1-pentene (APE), 6-amino1-hexene (AHE), 5-amino-1-pentyne (APYN), and 6-amino1-hexyne (AHYN), were prepared to examine the effects of the size and flexibility of the alkyl chain on $\mathrm{IC}_{50}$ values. Among these compounds, APE showed the highest potency as an inhibitor, and was more potent than the parent BA, suggesting that the best fit distance between the $\mathrm{N}$-atom and the terminus C-atom is about $0.59 \mathrm{~nm}$. Since introduction of a triple bond resulted in higher $\mathrm{IC}_{50}$ values, flexibility of alkyl chain is necessary for the best fitting. To examine the substituent effects at the 4-position of cyclohexylamine, three

\section{A}
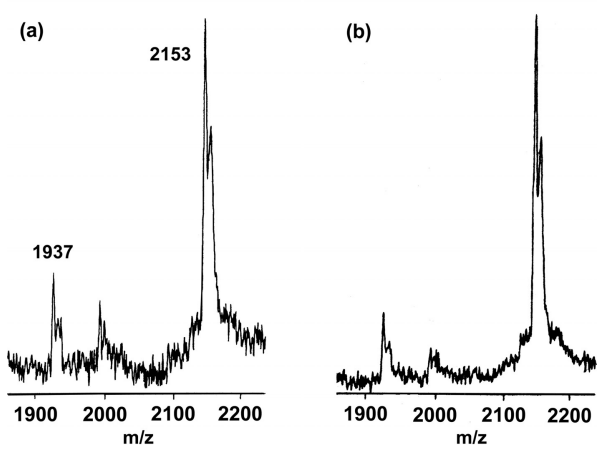

B
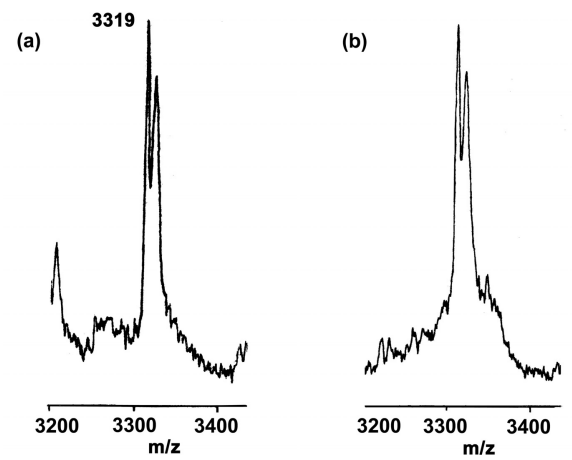

Fig. 5. MALDI Mass Spectra (Enlarged) over the Ranges of (A) $m / z$ $1900-2200$ and (B) $m / z 3200-3400$ in the (a) Presence and (b) Absence of DTT

Sample preparation and mass spectrometry were carried out as described in the legend of Fig. 3.
4MCHA analogs, 4,4-dimethylcyclohexylamine (DMCHA), 4-methylenecyclohexylamine (MLCHA), and trans-4-ethylcyclohexylamine (4ECHA), were prepared and tested. The analogs exhibited higher $\mathrm{IC}_{50}$ values than the parent 4MCHA. In particular, DMCHA did not show any inhibitory effects; presumably, its axial methyl group prevented the ana$\log$ from entering the active site.

\section{DISCUSSION}

Mammalian spd syn isolated from human, rat, and mouse, show over $96 \%$ identity in the amino acid sequence, and has ten common cysteine residues. ${ }^{4,5)}$ In contrast, T. maritima spd syn, $\left.{ }^{6}\right)$ which show $34 \%$ identity to rat spd syn, has three cysteine residues, of which only one matches $\mathrm{C} 123$ of rat spd syn in the sequence alignment. These primary sequence data suggest that a three dimensional model for mammalian spd syn can be based on the crystal structure of T. maritima spd syn, and that the determination of each cysteine residue of mammalian spd syn as sulfhydryl or disulfide will help refine the model. In the present study, the chemical determination of cysteine residues was restricted due to an insufficient denaturation of spd syn with guanidine in the absence of DTT for examining disulfide bond, as well as the small amount and easily oxidizable property of the purified enzyme. Therefore, the homology modeling approach was thought to be useful for estimating the three undetermined cysteine residues. A such model of rat spd syn (Fig. 6) was obtained from Swiss-Model protein modeling server (http://www.expasy.ch/swissmod/SWISS-MODEL.html), and does not include the N-terminal 26 and $\mathrm{C}$-terminal 3 amino acid residues, which did not align with the template sequence. Calculated distances between the three undetermined cysteine residues, C89, C236, and C251 (all shown in orange), indicate the unlikelihood of disulfide bonds between these residues. Although a disulfide bond between one of the three residues and $\mathrm{C} 25$ remain possible, it is unlikely that $\mathrm{C} 25$ can approach the residue without a large conformational change in the model. This model, therefore, suggests that the three cysteine residues are sulfhydryl. Consequently, a question arises as to the identity of the cysteine residue that serves as the counterpart to $\mathrm{C} 25$. Known crystal structures of bacterial spd syn that can form a dimer or tetramer may provide a hint to the answer. Bacterial N-terminal peptide regions corresponding to the rat $\mathrm{N}$-terminal 37 amino acids combine to themselves through $\beta$-hairpins in the functional oligomeric form (see PDB accession codes described above). Mammalian spd syn is a dimer, and therefore, the apparent role of

Table 1. $\mathrm{IC}_{50}$ Values of BA and 4MCHA Analogs

\begin{tabular}{|c|c|c|c|c|c|}
\hline BA analogs & $\mathrm{IC}_{50}(\mu \mathrm{M})$ & $\mathrm{N}-\mathrm{C}^{a)}(\mathrm{nm})$ & 4MCHA analogs & $\mathrm{IC}_{50}(\mu \mathrm{M})$ & $\mathrm{N}-\mathrm{C}^{a)}(\mathrm{nm})$ \\
\hline BA & 3.8 & 0.50 & 4MCHA & 1.7 & 0.58 \\
\hline PA & 3.6 & 0.63 & $c_{i s-\text { Isomer }^{b)}}$ & 430 & 0.48 \\
\hline HA & 105 & 0.75 & $\mathrm{CHA}$ & 17 & 0.43 \\
\hline $\mathrm{ABE}$ & 13.5 & 0.48 & 4ECHA & 135 & 0.67 \\
\hline APE & 1.7 & 0.59 & DMCHA & $>1000$ & $0.57(0.48)$ \\
\hline AHE & 22 & 0.72 & MLCHA & 60 & 0.54 \\
\hline APYN & 20 & 0.59 & 4HCHA & 85 & 0.56 \\
\hline AHYN & 40 & 0.73 & & & \\
\hline
\end{tabular}



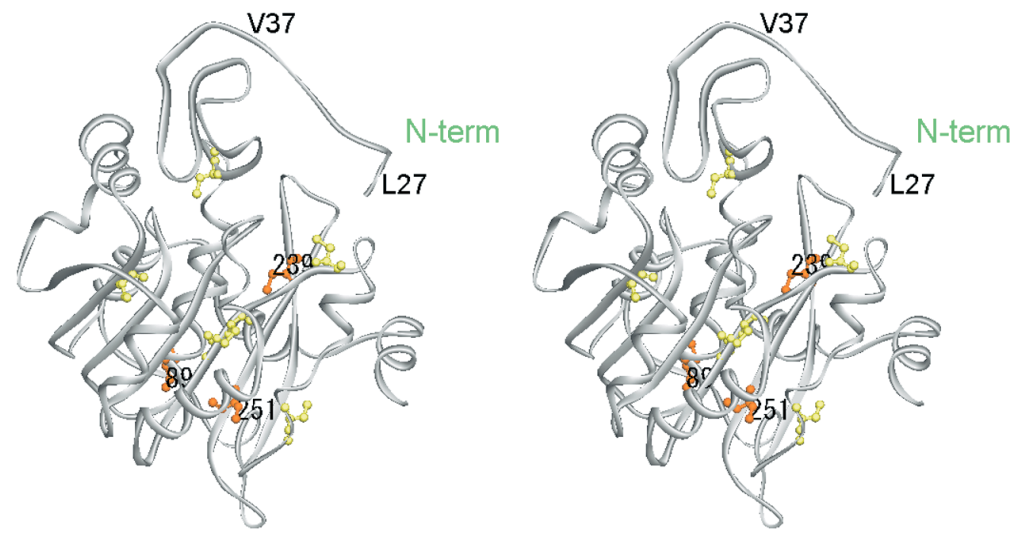

Fig. 6. Stereo-View of a Model of Rat Spd Syn

The model was based on the homologous T. maritima spd syn, complexed with AdoDATO (PDB accession code 1JQ3). The cysteine residues are shown in the ball and stick model; the three undetermined and six determined residues are shown in orange and yellow, respectively. N-terminus 26 and the three residues of the C-terminus are missing. All structural figures are displayed with DS ViewerPro (Accelrys Inc.)
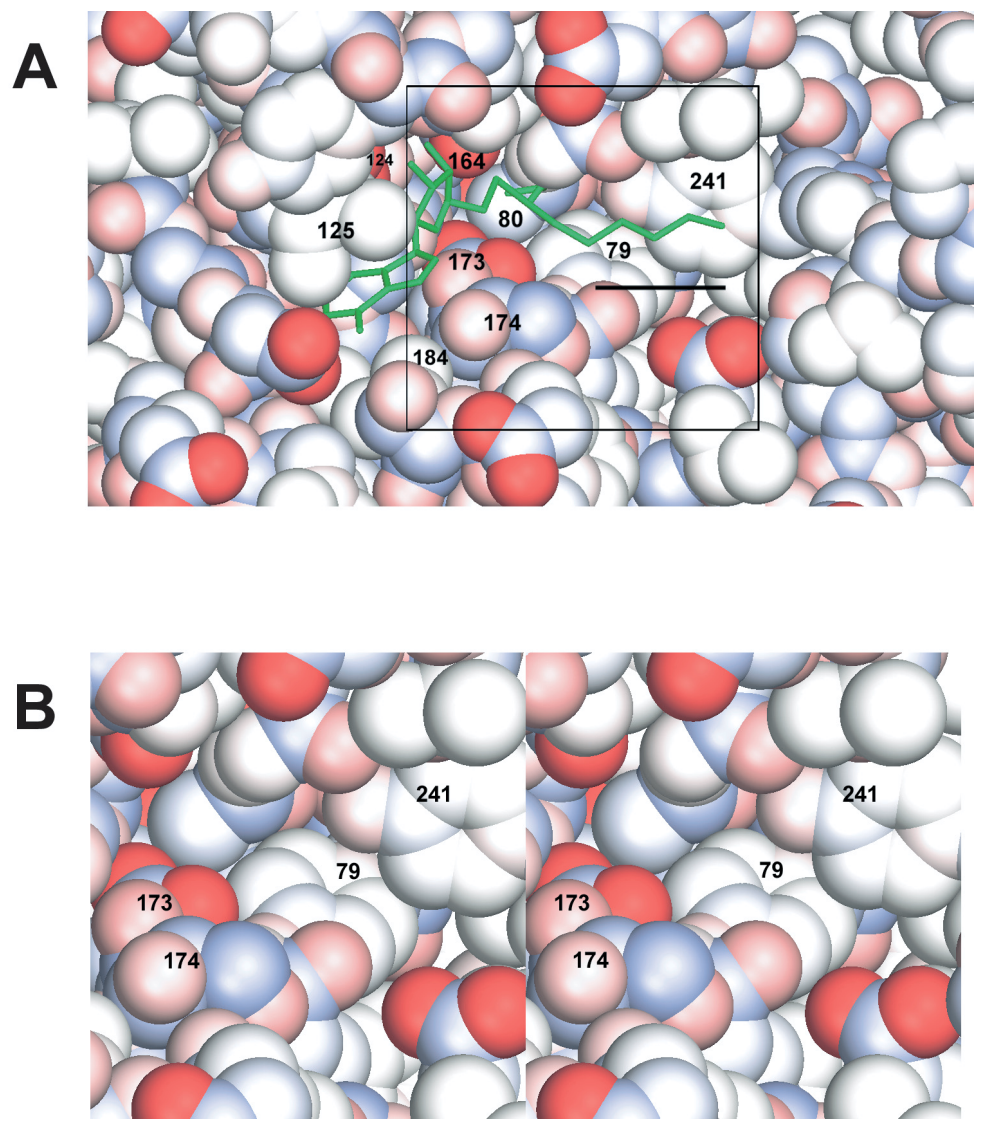

Fig. 7. Active Site in the Proposed 3-D Structure of Rat Spd Syn, Shown Using CPK Representation

Residues S175-A181, corresponding to the "gatekeeping" loop, are not shown. The partial charge of each atom is indicated as a gradient from red (negative) to blue. (A) 2-D view of the active site including both dcAdoMet and putrescine-binding site using AdoDATO (green) based on T. maritima spd syn. The length of bar inserted near putrescine binding site is $0.5 \mathrm{~nm}$. (B) enlarged 3-D view of the putrescine-binding site corresponding to the enclosed square area in (A).

the N-terminal peptide regions might be to reinforce the binding between the two protomers, although the binding need not be through $\beta$-hairpin. Since $\mathrm{C} 25$ is located within this region, it will be possible to suggest the existence of a disulfide bond between the two $\mathrm{C} 25$ residues of the protomers in formation of the dimer. Unfortunately, isolation of a peptide fragment containing such disulfide bond remains elusive, and therefore, the possibility of a disulfide bond be- tween C25 and a small sulfhydryl compound cannot be excluded at the present.

As shown using CPK representation (Fig. 7), the amino acid residues in the active site of T. maritima spd syn show good agreement to that of rat spd syn, i.e. (rat in parenthesis) Y76 (Y79), H77 (Q80), D101 (D104), E121 (E124), V122 (I125), D170 (D173), S171 (S174), D173 (D176), L182 (L184), and Y239 (Y241). The residues near the putrescine 
binding site in rat are Y79, D173, S174, D176, and Y241. D173 (D176) is the negatively charged site that can bind to the protonated amine group of putrescine, and is located in the "gatekeeping" loop" which is also present in rat enzyme. The "gatekeeping" loop is dynamic and serves to cover putrescine substrate. In the model, the "gatekeeping" loop is removed to illustrate the narrow groove that would accommodate the methylene group of putrescine. Based on the inhibitory activities of the BA and 4MCHA analogs, it is reasonable to define the compounds that enter this groove and inhibit spd syn activity as possessing a primary amine group, a flexible structure, and a distance of about $0.6 \mathrm{~nm}$ between the $\mathrm{N}$-atom and terminus $\mathrm{C}$-atom. Accordingly, the $\mathrm{N}-\mathrm{C}$ distance of APE, a novel potent inhibitor, is $0.59 \mathrm{~nm}$, which is similar to that of 4MCHA.

The presence of the "gatekeeping" loop indicates the dynamic nature of the putrescine-binding site, hence the difficulty in defining the best fitting compound into the site. In connection with the active site, different levels of fluorescence-labeling using IAEDANS were applied to rat spd syn in the presence and absence of dcAdoMet. Since the results indicate significant changes in the conformation of the enzyme when dcAdoMet is present in the active site, the model will be applied to dcAdoMet-binding spd syn.

Acknowledgements This work was supported in part by the Grant-in-Aid for Scientific Research (C) (2) (\# 13672261) from the Ministry of Education, Culture, Sports, Science and Technology, Japan. The authors express their deep gratitude to Professor Kozo Nagano, Tokyo Metropolitan Institute of Gerontology, for his kind suggestions.

\section{REFERENCES}

1) Marton L. J., Pegg A. E., Annu. Rev. Pharmacol. Toxicol., 35, 55-91 (1995).

2) Davis R. H., Morris D. R., Coffino P., Microbiol. Rev., 56, 280-290 (1992).

3) Seiler N., Delcros J. G., Moulinoux J. P., Int. J. Biochem. Cell Biol., 28 , 843-861 (1996).

4) Pegg A. E., Poulin R., Coward J. K., Int. J. Biochem. Cell Biol., 27, 425-442 (1995).

5) Hamasaki-Katagiri N., Tabor C. W., Tabor H., Gene, 187, 35-43 (1997).

6) Korolev S., Ikeguchi Y., Skarina T., Beasley S., Arrowsmith C., Edwards A., Joachimiak A., Pegg A. E., Savchenko A., Nat. Struct. Biol., 9, 27-31 (2002).

7) Wada M., Amano D., Hosoda H., Shirahata A., Samejima K., Pegg A. E., Biol. Pharm. Bull., 22, 889-895 (1999).

8) Shirahata A., Morohoshi T., Fukai M., Akatsu S., Samejima K., Biochem. Pharmacol., 41, 205-212 (1991).

9) Dejima H., Kobayashi M., Takasaki H., Takeda N., Shirahata A., Samejima K., Biol. Pharm. Bull., 26, 1005-1008 (2003).

10) Gagne M. R., Stern C. L., Marks T. J., J. Am. Chem. Soc., 114, $275-$ 294 (1992).

11) Mitsunobu O., Synthesis, 1981, 1-28 (1981).

12) Bordwell F. G., Wellman K. M., J. Org. Chem., 28, 1347-1352 (1963).

13) Johnston T. P., McCaleb G. S., Opliger, P. S., Laster W. R., Montgomery J. A., J. Med. Chem., 14, 600-614 (1971).

14) Frontier A. J., Danishefsky S. J., Koppel G. A., Meng D., Tetrahedron, 54, 12721-12736 (1998).

15) Samejima K., Raina A., Yamanoha B., Eloranta T., Methods Enzymol., 94, 270-276 (1983).

16) Hibasami H., Pegg A. E., Biochem. J., 169, 709-712 (1978).

17) Wu J., Gage D. A., Watson J. T., Anal. Biochem., 235, 161-174 (1996).

18) Russell W. K., Park Z.-Y., Russell D. H., Anal. Chem., 73, 2682-2685 (2001).

19) Masaki T., Fujihashi T., Nakamura K., Soejima M., Biochem. Biophys. Acta, 660, 51-55 (1981).

20) Coward J. K., Motola N. C., Moyer J. D., J. Med. Chem., 20, 500-505 (1977).

21) Liu C., Coward J. K., J. Med. Chem., 34, 2094-2101 (1991). 